\title{
Effects of etchants in the transfer of chemical vapor deposited graphene
}

\author{
M. Wang, ${ }^{1,2,3, a)}$ E. H. Yang, ${ }^{1}$ R. Vajtai, ${ }^{2}$ J. Kono, ${ }^{2,3,4}$ and P. M. Ajayan ${ }^{2}$ \\ ${ }^{1}$ Mechanical Engineering Department, Stevens Institute of Technology, Hoboken, New Jersey 07030, USA \\ ${ }^{2}$ Department of Materials Science and NanoEngineering, Rice University, Houston, Texas 77005, USA \\ ${ }^{3}$ Department of Electrical and Computer Engineering, Rice University, Houston, Texas 77005, USA \\ ${ }^{4}$ Department of Physics and Astronomy, Rice University, Houston, Texas 77005, USA
}

(Received 13 October 2017; accepted 17 March 2018; published online 18 May 2018)

\begin{abstract}
The quality of graphene can be strongly modified during the transfer process following chemical vapor deposition (CVD) growth. Here, we transferred CVD-grown graphene from a copper foil to a $\mathrm{SiO}_{2} / \mathrm{Si}$ substrate using wet etching with four different etchants: $\mathrm{HNO}_{3}, \mathrm{FeCl}_{3},\left(\mathrm{NH}_{4}\right)_{2} \mathrm{~S}_{2} \mathrm{O}_{8}$, and a commercial copper etchant. We then compared the quality of graphene after the transfer process in terms of surface modifications, pollutions (residues and contaminations), and electrical properties (mobility and density). Our tests and analyses showed that the commercial copper etchant provides the best structural integrity, the least amount of residues, and the smallest doping carrier concentration. Published by AIP Publishing. https://doi.org/10.1063/1.5009253
\end{abstract}

\section{INTRODUCTION}

Monolayer graphene, successfully isolated in 2004 for the first time, is the first member of the class of the so-called two-dimensional (2D) materials. It consists of a 2D honeycomb lattice of $\mathrm{sp}^{2}$-bonded carbon atoms and possesses extraordinary mechanical, chemical, and physical properties. Among all the methods of producing graphene, ${ }^{1-6}$ chemical vapor deposition (CVD) on metal surfaces, such as $\mathrm{Cu}, \mathrm{Ni}$, $\mathrm{Pt}, \mathrm{Ru}$, and $\mathrm{Ir}^{7}{ }^{7}$ is one of the most developed techniques to enable large-size, high-quality, and inexpensive growth. ${ }^{8}$ However, for characterization and electrical applications, it is usually necessary to transfer the CVD-grown graphene sheet onto another substrate. ${ }^{9}$ The common method for transferring is based on a wet etching process, which involves depositing a polymer, typically poly(methyl methacrylate) (PMMA) as a supporting layer, followed by etching of the metal substrate, and finishing with the deposition onto a desired substrate and subsequent removal of the polymer. In this process, regardless of the particular transfer method, graphene properties can be altered due to surface modifications ${ }^{10}$ residual polymer impurities, ${ }^{11,12}$ solution doping, ${ }^{13}$ and metallic contaminations (copper and iron). ${ }^{3,14}$ A large body of research work has been dedicated to minimizing these modifications; examples of such efforts include thermal annealing, electrical current annealing, plasma cleaning, chloroform treatment, and using a sacrificial Ti layer. Yet, these methods can still introduce defects in the graphene sheets or increase toxicity, ${ }^{7,15-17}$ and so, techniques to improve graphene quality are still strongly desired. On the other hand, methods to determine the quality of transferred graphene have been developing quickly, such as inductively coupled plasma mass spectrometry, electron energy loss spectroscopy, and X-ray photoelectron spectroscopy (XPS). ${ }^{7,20}$ For instance, total reflection X-ray fluorescence measurements are used for the determination of the trace and concentration of residual metals, ${ }^{14}$ Raman spectroscopy is

\footnotetext{
a) Author to whom correspondence should be addressed: janewangmj@ gmail.com
}

commonly used for monitoring dopants, ${ }^{11,21,22}$ and terahertz (THz) emission spectroscopy is utilized for imaging molecular adsorption on graphene. ${ }^{23} \mathrm{THz}$ time-domain spectroscopy (TDS) has also emerged as a sensitive and nondestructive technique that can determine the carrier density and mobility of graphene. ${ }^{24,25}$

Here, we investigate graphene layers grown by CVD on $\mathrm{Cu}$ foils and transferred onto $\mathrm{SiO}_{2} / \mathrm{Si}$ substrates by using four different types of etchants. We utilized TDS together with Raman spectroscopy and gated field effect transistor (FET) measurements to determine the most adequate etchant which causes the least contamination or doping due to the transfer process and accordingly results in the highest carrier mobility in graphene.

\section{EXPERIMENTAL DETAILS}

Large-area, monolayer graphene was synthesized on $\mathrm{Cu}$ via a CVD method reported by $\mathrm{Li}$ et al. ${ }^{7} \mathrm{~A} \mathrm{Cu}$ foil was placed in a hot region of the furnace kept at $1000{ }^{\circ} \mathrm{C}$, while the $\mathrm{Ar} / \mathrm{H}_{2}$ gas mixture was applied as a carrier and reduction gas and $\mathrm{CH}_{4}$ was introduced a carbon source. As-grown graphene was transferred using PMMA as a supporting layer and wet etching the $\mathrm{Cu}$ substrate. Graphene $(1 \mathrm{~cm} \times 1 \mathrm{~cm})$ was transferred onto a $440-\mu$ m-thick lightly doped p-type silicon wafer $(5-10 \Omega \mathrm{cm})$ capped with a 300 -nm-thick $\mathrm{SiO}_{2}$ layer.

Four different types of etchants were used to dissolve $\mathrm{Cu}$ foil- $\mathrm{HNO}_{3}, \mathrm{FeCl}_{3},\left(\mathrm{NH}_{4}\right)_{2} \mathrm{~S}_{2} \mathrm{O}_{8}$, and a commercial $\mathrm{Cu}$ etchant (1001875130, Sigma-Aldrich). $\mathrm{NO}_{2}$ bubbles were produced in the transfer process when $\mathrm{HNO}_{3}\left(16 \mathrm{~mol} \cdot \mathrm{l}^{-1}\right)$ was used, and sometimes bubbles broke out from the graphene/PMMA film when the reaction was too intense; accordingly, a lower concentration of the $\mathrm{HNO}_{3}$ solution (1:8 diluted) was chosen to safely slow down the etching process. It took $4-6 \mathrm{~h}$ to completely remove the $\mathrm{Cu}$ foil from the graphene/PMMA film. The etching mechanism for $\mathrm{HNO}_{3}$ is described by $\mathrm{Cu}+\mathrm{HNO}_{3} \rightarrow \mathrm{Cu}\left(\mathrm{NO}_{3}\right)_{2}+\mathrm{NO}_{2}$. $\mathrm{FeCl}_{3}$ is a safer choice than $\mathrm{HNO}_{3}$ since no gas was produced in the reaction. We used $10 \% \mathrm{FeCl}_{3}$ solution, and the etching time was as short as 10 min without causing significant surface modifications and 
damages to graphene. The etching mechanism for $\mathrm{FeCl}_{3}$ is described by $\mathrm{FeCl}_{3}+\mathrm{Cu} \rightarrow \mathrm{FeCl}_{2}+\mathrm{CuCl}, \quad \mathrm{FeCl}_{3}+\mathrm{CuCl}$ $\rightarrow \mathrm{FeCl}_{2}+\mathrm{CuCl}_{2}$, and $\mathrm{CuCl}_{2}+\mathrm{Cu} \rightarrow \mathrm{CuCl}$. $\left(\mathrm{NH}_{4}\right)_{2} \mathrm{~S}_{2} \mathrm{O}_{8}$ is another type of etchant, prevailing over avoiding metallic contamination. It took $1-2 \mathrm{~h}$ to dissolve the $\mathrm{Cu}$ foil at a concentration of $0.1 \mathrm{M}$. The etching mechanism for $\left(\mathrm{NH}_{4}\right)_{2} \mathrm{~S}_{2} \mathrm{O}_{8}$ is described by $\mathrm{Cu}+\left(\mathrm{NH}_{4}\right)_{2} \mathrm{~S}_{2} \mathrm{O}_{8} \rightarrow \mathrm{CuSO}_{4}+\left(\mathrm{NH}_{4}\right)_{2} \mathrm{SO}_{4}$, $\left(\mathrm{NH}_{4}\right)_{2} \mathrm{~S}_{2} \mathrm{O}_{8}+\mathrm{H}_{2} \mathrm{O} \rightarrow \mathrm{H}_{2} \mathrm{SO}_{4}+\left(\mathrm{NH}_{4}\right)_{2} \mathrm{SO}_{4}+(\mathrm{O}), \quad$ and $\mathrm{Cu}+(\mathrm{O})+\mathrm{H}_{2} \mathrm{SO}_{4} \rightarrow \mathrm{CuSO}_{4}+\mathrm{H}_{2} \mathrm{O}$. Finally, a commercial $\mathrm{FeCl}_{3}$-based $\mathrm{Cu}$ etchant (1001875130, Sigma-Aldrich) was also used for etching the $\mathrm{Cu}$ foil.

\section{RESULTS AND DISCUSSION}

Figure 1 shows optical microscopy images of graphene transferred onto $\mathrm{SiO}_{2} / \mathrm{Si}$ substrates using (a) $\mathrm{HNO}_{3}$, (b) $\mathrm{FeCl}_{3}$, (c) $\left(\mathrm{NH}_{4}\right)_{2} \mathrm{~S}_{2} \mathrm{O}_{8}$, and (d) the $\mathrm{Cu}$ etchant. Winkles were observed in all of these samples, but $\left(\mathrm{NH}_{4}\right)_{2} \mathrm{~S}_{2} \mathrm{O}_{8}$ and the $\mathrm{Cu}$ etchant led to clean surfaces, having fewer spots of residues when observed through optical microscopy. $\mathrm{FeCl}_{3}$ and the $\mathrm{Cu}$ etchant, two etchants that both have $\mathrm{Fe}^{3+}$ as the active ingredient, led to more continuous morphologies of graphene with lower amounts of cracks and holes. This could be understood by analyzing the content of each etchant: graphene transferred with $\mathrm{HNO}_{3}$ had the largest number of holes due to $\mathrm{NO}_{2}$ bubbles produced in etching, as described above; cracks and holes are also observed in graphene transferred with $\left(\mathrm{NH}_{4}\right)_{2} \mathrm{~S}_{2} \mathrm{O}_{8}$ because $\left(\mathrm{NH}_{4}\right)_{2} \mathrm{~S}_{2} \mathrm{O}_{8}$ has remarkable strong oxidizing capacity. The standard electrode potential of $\mathrm{S}_{2} \mathrm{O}_{8}{ }^{2-}$ is as high as $2.05 \mathrm{~V}$, which is almost three times of that of $\mathrm{Fe}^{3+}, 0.77 \mathrm{~V}$. So, $\mathrm{S}_{2} \mathrm{O}_{8}{ }^{2-}$ can oxidize and damage the protecting PMMA layer, and cracks and holes were then made. $\mathrm{Fe}^{3+}$ base solutions are mild and safer, and the commercial $\mathrm{Cu}$ etchant, which contains a wetting anti-foam agent and can provide a very stable etch rate of $0.5 \mathrm{mil} / \mathrm{min}$ at $40^{\circ} \mathrm{C}$, can further improve the integrity and reduce polymer residues. Therefore, as far as the morphology is concerned, the commercial $\mathrm{Cu}$ etchant was found to provide the cleanest and most undamaged graphene after transfer.

It is known that the $\mathrm{G}$ and $2 \mathrm{D}$ peaks shift as a function of doping in graphene. ${ }^{11,22}$ In addition, these peaks have different doping dependences, and thus, the 2D/G intensity ratio changes significantly with doping, making it a sensitive parameter to monitor the doping level. The exact position of each peak slightly varied from sample to sample, and so, a large number of samples that were grown and transferred at the same time were studied, and the average values of each peak position were used for further calculations. Typical Raman spectra in the G (left) and 2D (right) regions for graphene samples transferred onto $\mathrm{SiO}_{2} / \mathrm{Si}$ substrates with the four etchants are shown in Fig. 2. The black, red, green, and blue lines correspond to $\mathrm{HNO}_{3}, \mathrm{FeCl}_{3},\left(\mathrm{NH}_{4}\right)_{2} \mathrm{~S}_{2} \mathrm{O}_{8}$, and the commercial $\mathrm{Cu}$ etchant, respectively.

Table I lists the average frequencies of the $\mathrm{G}$ and 2D peaks and their corresponding electron densities calculated from the reported peak frequency-doping relationship. ${ }^{22}$ The Fermi energy, $E_{\mathrm{F}}$, of graphene can be calculated through $E_{\mathrm{F}}= \pm \hbar v_{\mathrm{F}}(\pi|n|)^{1 / 2}$, where $v_{\mathrm{F}} \approx 10^{6} \mathrm{~m} / \mathrm{s}$ and $n$ is the carrier density. From the position of both peaks, it can be seen that $\mathrm{FeCl}_{3}$ and the $\mathrm{Cu}$ etchant, the two etchants that have $\mathrm{Fe}^{3+}$ as the active ingredient, provided lower charge densities than the other two etchants, indicating a lower doping capability. The commercial $\mathrm{Cu}$ etchant led to the smallest value, while $\mathrm{HNO}_{3}$ led to the largest value of carrier density. Both hetero atom doping and chemical modification need to be considered here. Metallic contaminations (copper and iron) were previously demonstrated to be introduced in transfer, ${ }^{14}$ as discussed above, and p-type doping by $\mathrm{HNO}_{3}$ was also
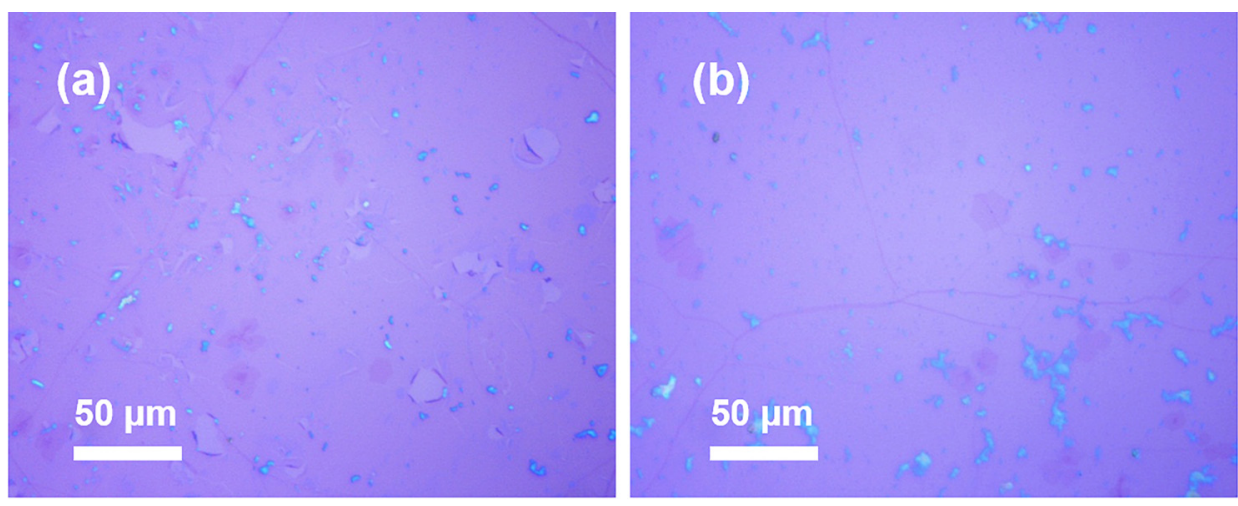

(c)

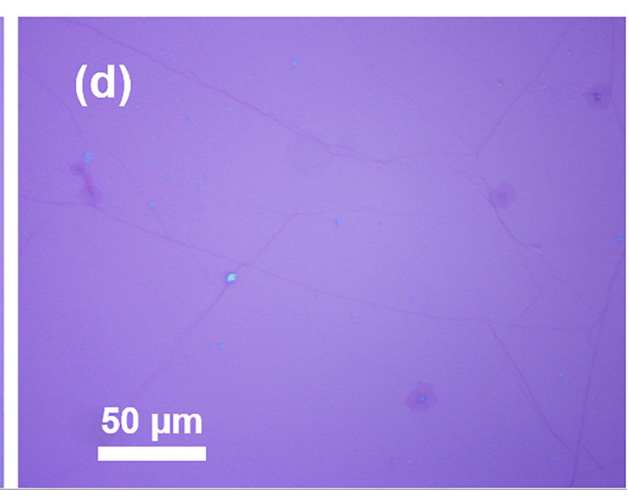

FIG. 1. Optical microscopy images of graphene transferred onto $\mathrm{SiO}_{2} / \mathrm{Si}$ substrates by wet etching with (a) $\mathrm{HNO}_{3}$, (b) $\mathrm{FeCl}_{3}$, (c) $\left(\mathrm{NH}_{4}\right)_{2} \mathrm{~S}_{2} \mathrm{O}_{8}$, and (d) the $\mathrm{Cu}$ etchant. 


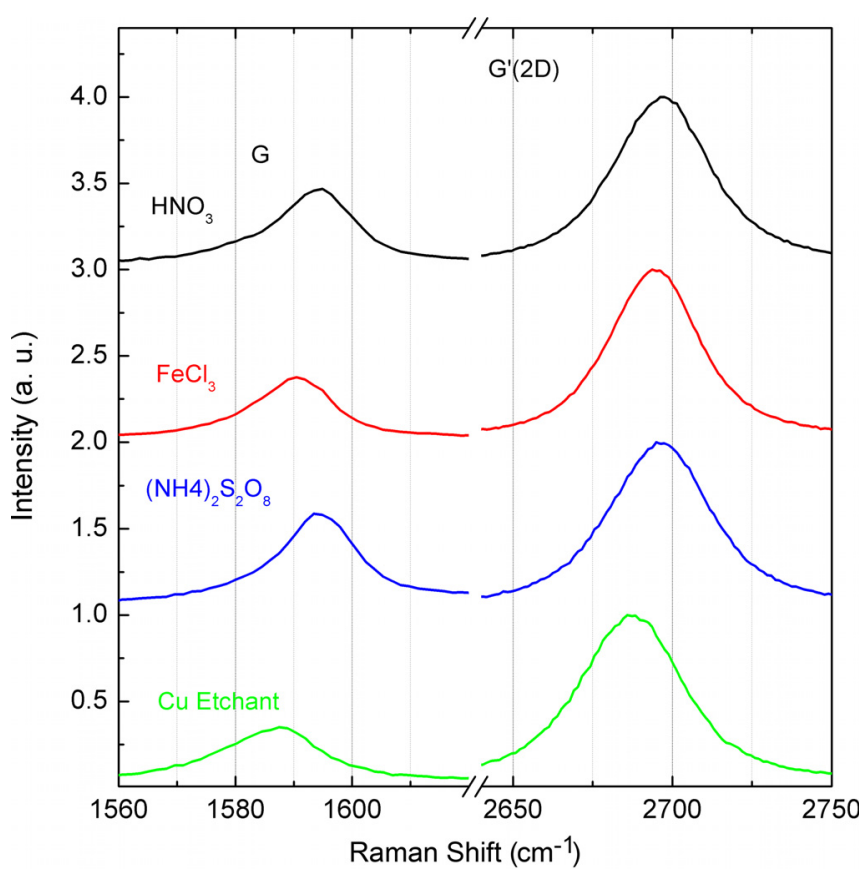

FIG. 2. Raman spectra for graphene samples transferred onto $\mathrm{SiO}_{2} / \mathrm{Si}$ substrates using wet etching with $\mathrm{HNO}_{3}, \mathrm{FeCl}_{3},\left(\mathrm{NH}_{4}\right)_{2} \mathrm{~S}_{2} \mathrm{O}_{8}$, and the $\mathrm{Cu}$ etchant.

confirmed to be stable. ${ }^{26}$ Furthermore, adsorbed molecules such as $\mathrm{NO}_{2}$, which is produced in the etching process by $\mathrm{HNO}_{3}$, can also change the local carrier concentration in graphene. ${ }^{27}$ Interestingly and reasonably, although $\mathrm{FeCl}_{3}$ caused more visible residues on the surface, as observed under an optical microscope, than $\left(\mathrm{NH}_{4}\right)_{2} \mathrm{~S}_{2} \mathrm{O}_{8}$, graphene transferred with it actually had a lower doping level, implying lower level contamination in graphene. This can be explained since sulfuric acid molecules produced in the etching process by $\left(\mathrm{NH}_{4}\right)_{2} \mathrm{~S}_{2} \mathrm{O}_{8}$ are also reported to be absorbed by graphene. ${ }^{28}$ Fermi levels for all the samples obtained here are on the order of several hundreds of meV. These values agree with the previously reported values for CVD-grown and transferred graphene. ${ }^{29}$ Moreover, the D peak is known by its dependence on disorder such as edges and defects in graphene, ${ }^{30}$ and graphene transferred with $\mathrm{HNO}_{3}$ and $\left(\mathrm{NH}_{4}\right)_{2} \mathrm{~S}_{2} \mathrm{O}_{8}$ does have stronger $\mathrm{D}$ peaks than those with $\mathrm{Fe}^{3+}$ based etchants (data not shown).

In combination with Raman spectroscopy measurements, DC electronic transport measurements were performed to electronically characterize graphene samples using a graphene/ $\mathrm{SiO}_{2} / \mathrm{p}-\mathrm{Si}$ FET. The minimum conductance point (Dirac Point), doping densities, Fermi energy, and Hall mobility $\mu=1 /$ enR [in units of $\mathrm{cm}^{2} /(\mathrm{V} \mathrm{s})$ ] were extracted from the

TABLE I. Raman peak positions and corresponding doping densities and mobilities for graphene transferred with different etchants.

\begin{tabular}{lccccc}
\hline \hline Etchant & $\begin{array}{c}\mathrm{G} \mathrm{Peak} \\
\left(\mathrm{cm}^{-1}\right)\end{array}$ & $\begin{array}{c}n_{G} \\
\left(10^{12} \mathrm{~cm}^{-2}\right)\end{array}$ & $\begin{array}{c}2 \mathrm{D} \text { peak } \\
\left(\mathrm{cm}^{-1}\right)\end{array}$ & $\begin{array}{c}n_{2 D} \\
\left(10^{12} \mathrm{~cm}^{-2}\right)\end{array}$ & $E_{\mathrm{F}}(\mathrm{meV})$ \\
\hline $\mathrm{HNO}_{3}$ & 1595 & 10 & 2697 & 18 & 406 \\
$\mathrm{FeCl}_{3}$ & 1588 & 5 & 2695 & 12 & 287 \\
$\left(\mathrm{NH}_{4}\right)_{2} \mathrm{~S}_{2} \mathrm{O}_{8}$ & 1593 & 8 & 2697 & 18 & 363 \\
$\mathrm{Cu} \mathrm{Etchant}$ & 1585 & 3 & 2689 & 4 & 203 \\
\hline \hline
\end{tabular}

TABLE II. Dirac point, carrier density, Fermi energy, resistance, and mobility for graphene transferred with different etchants from Raman spectroscopy.

\begin{tabular}{lcccc}
\hline \hline Etchant & $\begin{array}{c}\text { Dirac point } \\
(\mathrm{mV})\end{array}$ & $n_{F E T}\left(10^{12} \mathrm{~cm}^{-2}\right)$ & $\begin{array}{c}\text { Fermi } \\
\text { energy }(\mathrm{meV})\end{array}$ & $\begin{array}{c}\text { Mobility } \\
{\left[\mathrm{cm}^{2} /(\mathrm{V} \mathrm{s})\right]}\end{array}$ \\
\hline $\mathrm{HNO}_{3}$ & 132 & 9.2 & 390 & $\sim 5000$ \\
$\mathrm{FeCl}_{3}$ & 98 & 6.9 & 340 & $\sim 6000$ \\
$\left(\mathrm{NH}_{4}\right)_{2} \mathrm{~S}_{2} \mathrm{O}_{8}$ & 111 & 7.8 & 360 & $\sim 8000$ \\
$\mathrm{Cu} \mathrm{Etchant}$ & 76 & 5.3 & 290 & $\sim 9000$ \\
\hline \hline
\end{tabular}

$I_{\mathrm{d}}-V_{\mathrm{g}}$ curves using a method reported before ${ }^{17}$ and listed in Table II. The Fermi levels for all the samples obtained here are in the range of $200-400 \mathrm{meV}$, and carrier densities are on the order of $10^{12} \mathrm{~cm}^{-2}$. Relatively speaking, $\mathrm{FeCl}_{3}$ and the $\mathrm{Cu}$ etchant, the two etchants that have $\mathrm{Fe}^{3+}$ as the active ingredient, correspond to the lowest charge densities. The commercial $\mathrm{Cu}$ etchant leads to the smallest value, while $\mathrm{HNO}_{3}$ leads to the largest value of charge density. All these results agree with the results from Raman spectroscopy. The value of mobility of graphene transferred with different solutions increases on the order of $\mathrm{HNO}_{3}, \mathrm{FeCl}_{3},\left(\mathrm{NH}_{4}\right)_{2} \mathrm{~S}_{2} \mathrm{O}_{8}$, and the $\mathrm{Cu}$ etchant.

A commercial TDS system (Advantest, TAS7500TS) and a conventional Fourier transform infrared (FTIR) spectrometer (JASCO FT/IR-660 Plus) were used to measure the optical conductivity of the large-area graphene samples $(1 \mathrm{~cm} \times 1 \mathrm{~cm})$. By combining TDS and FTIR with different types of beam splitters and detectors, we obtained spectra in a frequency range from $6 \mathrm{~cm}^{-1}$ to $10000 \mathrm{~cm}^{-1}$. The transmittance spectrum $T(\omega)$ of graphene was obtained by rationing the transmitted signal through the graphene/substrate sample to that obtained for a reference $\mathrm{SiO}_{2} / \mathrm{Si}$ substrate with the same thickness. The transmittance is defined as $T(\omega)=\left|\tilde{E}_{s}(\omega) / \tilde{E}_{r}(\omega)\right|^{2}$, where $\tilde{E}_{s}$ and $\tilde{E}_{r}$ are the complex $\mathrm{THz}$ signals in the frequency domain after Fourier transformation from their time-domain data for the graphene/substrate sample and reference $\left(\mathrm{SiO}_{2} / \mathrm{Si}\right)$, respectively. The real part of graphene s $2 \mathrm{D}$ sheet complex conductivity $\sigma^{\prime}(\omega)$ was obtained from the following equation:

$$
T(\varpi)=\left(1+\frac{\pi \alpha}{1+n_{\text {sub }}} \frac{\sigma^{\prime}(\omega)}{\frac{\pi e^{2}}{2 h}}\right)^{-2},
$$

where $\alpha$ is the fine structure constant $(=1 / 137), n_{\text {sub }}$ is the refractive index of the substrate, $e$ is the electronic charge, and $h$ is the Planck constant. The experimental data $\sigma^{\prime}(\omega)$ can then be fit with the Drude model to deduce the carrier density and mobility using

$$
\tilde{\sigma}(\varpi)=\frac{\sigma_{0}}{1-i \varpi \tau},
$$

where $\sigma_{0}$ is the DC conductivity and $\tau$ is the carrier scattering time. A typical real part of conductivity for graphene transferred with the $\mathrm{Cu}$ etchant was fitted and shown in Fig. 3.

The deduced values are listed in Table III. Carrier densities, which are on the order of $10^{12} \mathrm{~cm}^{-2}$ for all the samples obtained, are close to the values obtained from Raman spectroscopy and DC transport measurements and have the same 


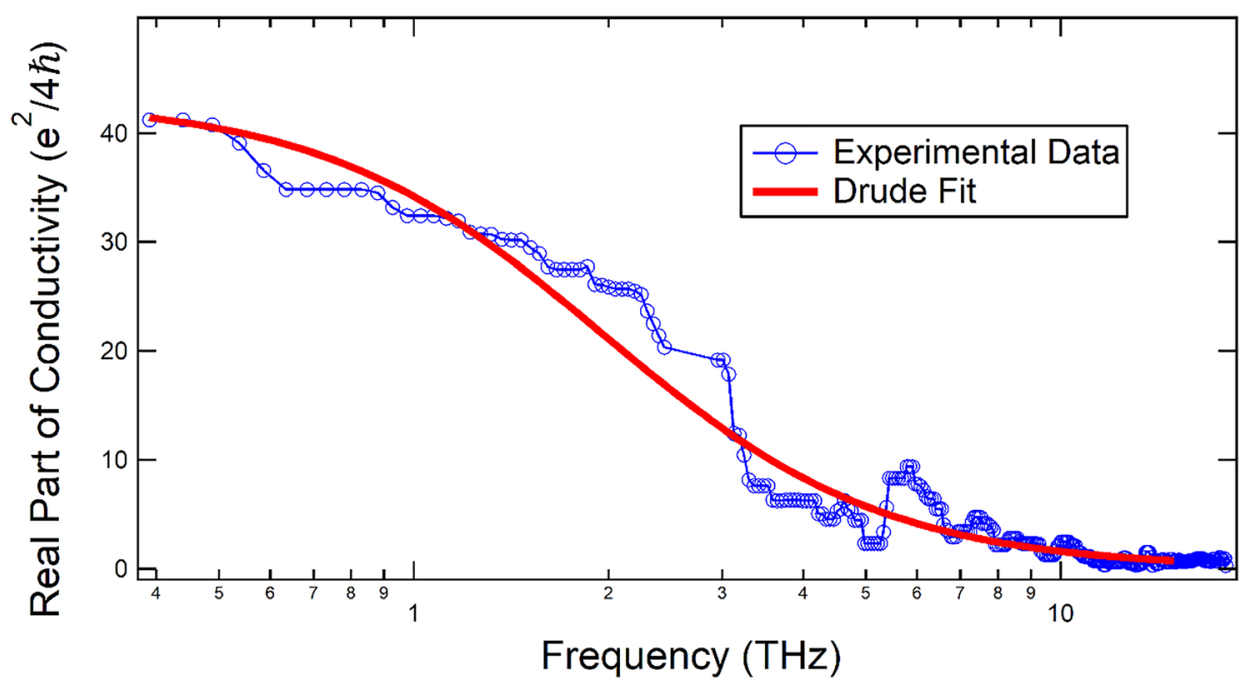

FIG. 3. Typical Drude model fitting for the optical conductivity of graphene transferred with a $\mathrm{Cu}$ etchant on a $\mathrm{SiO}_{2} / \mathrm{p}-\mathrm{Si}$ substrate in the $\mathrm{THz}$ and IR ranges. Open circles are the experimental data, and the solid line is the Drude fitting curve.

TABLE III. DC conductivity, carrier density, Fermi energy, resistance, and mobility for graphene transferred with different etchants subtracted from $\mathrm{THz}$ and FTIR data.

\begin{tabular}{lcccc}
\hline \hline Etchant & $\begin{array}{c}\text { DC conductivity } \\
\left(2 \pi e^{2} / \mathrm{h}\right)\end{array}$ & $\begin{array}{c}\text { Scattering } \\
\text { time }(\mathrm{ps})\end{array}$ & $\begin{array}{c}n_{\mathrm{THz}} \\
\left(10^{12} \mathrm{~cm}^{-2}\right)\end{array}$ & $\begin{array}{c}\text { Mobility } \\
{\left[\mathrm{cm}^{2} /(\mathrm{V} \mathrm{s})\right]}\end{array}$ \\
\hline $\mathrm{HNO}_{3}$ & 23 & 15 & 10 & 400 \\
$\mathrm{FeCl}_{3}$ & 28 & 18 & 5 & 690 \\
$(\mathrm{NH} 4)_{2} \mathrm{~S}_{2} \mathrm{O}_{8}$ & 34 & 14 & 8 & 420 \\
$\mathrm{Cu}$ Etchant & 33 & 29 & 3 & 2100 \\
\hline \hline
\end{tabular}

trend among the four different etchants: The value of carrier density of graphene transferred with different solutions increases in the order of $\mathrm{HNO}_{3},\left(\mathrm{NH}_{4}\right)_{2} \mathrm{~S}_{2} \mathrm{O}_{8}, \mathrm{FeCl}_{3}$, and the $\mathrm{Cu}$ etchant.

\section{CONCLUSIONS}

In summary, graphene samples grown by the CVD method on $\mathrm{Cu}$ foil were transferred onto $\mathrm{SiO}_{2} / \mathrm{p}$-Si substrates using a method including a wet etching step, and we characterized them without further processing, such as baking or annealing. Optical microscopy, Raman spectroscopy, DC transport, and THz-FTIR measurements were used to examine the quality of the transferred graphene, and we found that these kinds of the etchants have an effect on the quality of graphene. Four types of etchants were investigated, $\mathrm{HNO}_{3}, \mathrm{FeCl}_{3},\left(\mathrm{NH}_{4}\right)_{2} \mathrm{~S}_{2} \mathrm{O}_{8}$, and a commercial $\mathrm{Cu}$ etchant, and we concluded that the $\mathrm{Cu}$ etchant had the best performance for graphene transfer in this protocol since it leads to the best structural integrity, the least surface residue, the smallest doping carrier concentration, the highest mobility, and the longest scattering time.

\section{SUPPLEMENTARY MATERIAL}

See supplementary material for the detailed protocol used for graphene transfer and the detailed protocol used for photolithography for $\mathrm{SiO}_{2} / \mathrm{p}-\mathrm{Si}$ FET fabrication.

${ }^{1}$ P. Avouris, Nano Lett. 10, 4285 (2010)

${ }^{2}$ A. K. Geim and K. S. Novoselov, Nat. Mater. 6, 183 (2007).

${ }^{3}$ K. S. Novoselov, A. K. Geim, S. V. Morozov, D. Jiang, M. I. Katsnelson, I. V. Grigorieva, S. V. Dubonos, and A. A. Firsov, Nature 438, 197 (2005).
${ }^{4}$ K. S. Novoselov, A. K. Geim, S. V. Morozov, D. Jiang, Y. Zhang, S. V. Dubonos, I. V. Grigorieva, and A. A. Firsov, Science 306, 666 (2004).

${ }^{5}$ L. B. Tang, X. M. Li, R. B. Ji, K. S. Teng, G. Tai, J. Ye, C. S. Wei, and S. P. Lau, J. Mater. Chem. 22, 5676 (2012).

${ }^{6}$ Y. B. Zhang, Y. W. Tan, H. L. Stormer, and P. Kim, Nature 438, 201 (2005).

${ }^{7}$ X. S. Li, Y. W. Zhu, W. W. Cai, M. Borysiak, B. Y. Han, D. Chen, R. D. Piner, L. Colombo, and R. S. Ruoff, Nano Lett. 9, 4359 (2009).

${ }^{8}$ Y. Zhang, L. Y. Zhang, and C. W. Zhou, Acc. Chem. Res. 46, 2329 (2013).

${ }^{9}$ J. Leclercq and P. Sveshtarov, Bulg. J. Phys. 43, 121 (2016), http:// www.bjp-bg.com/papers/bjp2016_2_121-147.pdf.

${ }^{10}$ Y. Chen, X. L. Gong, and J. G. Gai, Adv. Sci. 3, 1500343 (2016).

${ }^{11}$ K. Kumar, Y. S. Kim, and E. H. Yang, Carbon 65, 35 (2013).

${ }^{12}$ J. W. Suk, W. H. Lee, J. Lee, H. Chou, R. D. Piner, Y. F. Hao, D. Akinwande, and R. S. Ruoff, Nano Lett. 13, 1462 (2013).

${ }^{13}$ L. D'Arsie, S. Esconjauregui, R. S. Weatherup, X. Y. Wu, W. E. Arter, H. Sugime, C. Cepek, and J. Robertson, RSC Adv. 6, 113185 (2016).

${ }^{14}$ G. Lupina, J. Kitzmann, I. Costina, M. Lukosius, C. Wenger, A. Wolff, S. Vaziri, M. Ostling, I. Pasternak, A. Krajewska, W. Strupinski, S. Kataria, A. Gahoi, M. C. Lemme, G. Ruhl, G. Zoth, O. Luxenhofer, and W. Mehr, ACS Nano 9, 4776 (2015).

${ }^{15}$ J. J. Ding, K. Du, I. Wathuthanthri, C. H. Choi, F. T. Fisher, and E. H. Yang, J. Vac. Sci. Technol. B 32, 06FF01 (2014).

${ }^{16}$ C. A. Joiner, T. Roy, Z. R. Hesabi, B. Chakrabarti, and E. M. Vogel, Appl. Phys. Lett. 104, 223109 (2014).

${ }^{17}$ V. Patil, A. Capone, S. Strauf, and E. H. Yang, Sci. Rep. 3, 2791 (2013).

${ }^{18}$ B. Aleman, W. Regan, S. Aloni, V. Altoe, N. Alem, C. Girit, B. S. Geng, L. Maserati, M. Crommie, F. Wang, and A. Zettl, ACS Nano 4, 4762 (2010).

${ }^{19}$ A. Ambrosi, C. K. Chua, B. Khezri, Z. Sofer, R. D. Webster, and M. Pumera, Proc. Natl. Acad. Sci. U.S.A. 109, 12899 (2012).

${ }^{20}$ A. Ambrosi and M. Pumera, Nanoscale 6, 472 (2014).

${ }^{21}$ S. M. Choi, S. H. Jhi, and Y. W. Son, Phys. Rev. B 81, 081407 (2010).

${ }^{22}$ A. Das, S. Pisana, B. Chakraborty, S. Piscanec, S. K. Saha, U. V. Waghmare, K. S. Novoselov, H. R. Krishnamurthy, A. K. Geim, A. C. Ferrari, and A. K. Sood, Nat. Nanotechnol. 3, 210 (2008).

${ }^{23}$ Y. Sano, I. Kawayama, M. Tabata, K. A. Salek, H. Murakami, M. Wang, R. Vajtai, P. M. Ajayan, J. Kono, and M. Tonouchi, Sci. Rep. 4, 6046 (2014).

${ }^{24}$ X. L. Liang, B. A. Sperling, I. Calizo, G. J. Cheng, C. A. Hacker, Q. Zhang, Y. Obeng, K. Yan, H. L. Peng, Q. L. Li, X. X. Zhu, H. Yuan, A. R. H. Walker, Z. F. Liu, L. M. Peng, and C. A. Richter, ACS Nano 5, 9144 (2011).

${ }^{25}$ L. Ren, Q. Zhang, J. Yao, Z. Z. Sun, R. Kaneko, Z. Yan, S. Nanot, Z. Jin, I. Kawayama, M. Tonouchi, J. M. Tour, and J. Kono, Nano Lett. 12, 3711 (2012).

${ }^{26}$ L. D'Arsié, S. Esconjauregui, R. S. Weatherup, X. Wu, W. E. Arter, H. Sugime, C. Cepek, and J. Robertson, RSC Adv. 6, 113185 (2016).

${ }^{27}$ F. Schedin, A. Geim, S. Morozov, E. Hill, P. Blake, M. Katsnelson, and K. Novoselov, Nat. Mater. 6, 652 (2007).

${ }^{28}$ I. G. Ayala, N. A. Cordero, and J. A. Alonso, Phys. Rev. B 84, 165424 (2011).

${ }^{29}$ L. Ren, Q. Zhang, S. Nanot, I. Kawayama, M. Tonouchi, and J. Kono, J. Infrared, Millimeter, Terahertz Waves 33, 846 (2012).

${ }^{30}$ R. Beams, L. G. Cancado, and L. Novotny, J. Phys.: Condens. Matter 27, 083002 (2015). 